\title{
The role of Candida albicans in root caries biofilms: an RNA-seq analysis
}

\section{Abstract}

Laís Daniela EV ${ }^{1}$

Nailê DAMÉ-TEIXEIRA ${ }^{2}$

Thuy $\mathrm{DO}^{3}$ iD

Marisa MALTZ ${ }^{1}$

Clarissa Cavalcanti Fatturi PAROLO ${ }^{1}$
Submitted: October 1, 2019 Modification: November 20, 2019

Accepted: January 19, 2020
Corresponding address: Laís Daniela Ev Departamento de Odontologia Preventiva e Social - Faculdade de Odontologia Universidade Federal do Rio Grande do Sul. Rua Ramiro Barcelos 2492 - Santa Cecília - 90035004 - Porto Alegre - RS - Brasil.

Phone: 555133085193 - Fax: 55133085023 e-mail: laisdanielaev@gmail.com
Objective: This study sought to analyze the gene expression of Candida albicans in sound root surface and root caries lesions, exploring its role in root caries pathogenesis. Methodology: The differential gene expression of C. albicans and the specific genes related to cariogenic traits were studied in association with samples of biofilm collected from exposed sound root surface (SRS, $n=10$ ) and from biofilm and carious dentin of active root carious lesions ( $R C, n=9$ ). The total microbial RNA was extracted, and the cDNA libraries were prepared and sequenced on the Illumina Hi-Seq2500. Unique reads were mapped to 163 oral microbial reference genomes including two chromosomes of C. albicans SC5314 (14,217 genes). The putative presence of $C$. albicans was estimated (sum of reads/total number of genes $\geq 1$ ) in each sample. Count data were normalized (using the DESeq method package) to analyze differential gene expression (using the DESeq2R package) applying the Benjamini-Hochberg correction (FDR $<0.05)$. Results: Two genes ( $\mathrm{CaO} 19.610, \mathrm{FDR}=0.009 ; \mathrm{CaO19.2506}$, FDR=0.018) were upregulated on SRS, and their functions are related to biofilm formation. Seven genes (UTP20, FDR $=0.018 ;$ ITR1, FDR $=0.036$; DHN6, FDR=0.046; $\mathrm{CaO} 19.7197, \mathrm{FDR}=0.046 ; \mathrm{CaO} 19.7838, \mathrm{FDR}=0.046 ; \mathrm{STT4}, \mathrm{FDR}=0.046$; GUT1, FDR =0.046) were up-regulated on RC and their functions are related to metabolic activity, sugar transport, stress tolerance, invasion and $\mathrm{pH}$ regulation. The use of alternative carbon sources, including lactate, and the ability to form hypha may be a unique trait of $C$. albicans influencing biofilm virulence. Conclusions: C. albicans is metabolically active in SRS and RC biofilm, with different roles in health and disease.

Keywords: Sequence analysis. RNA. Candida albicans. Root caries. Transcriptome.
'Universidade de Federal do Rio Grande do Sul, Faculdade de Odontologia, Departamento de Odontologia Preventiva e Social, Porto Alegre, Rio Grande do Sul, Brasil.

2Universidade de Brasília, Faculdade de Ciências da Saúde, Departamento de Odontologia, Brasília, Distrito Federal, Brasil.

${ }^{3}$ University of Leeds, Faculty of Medicine \& Health, School of Dentistry, Leeds, United Kingdom. 


\section{Introduction}

The bacterial biofilm associated with root caries lesions must harbor microorganisms that can produce acid from carbohydrates (acidogenicity) and must be able to growth in a low-pH environment (aciduricity). ${ }^{1}$ Diverse bacteria are prevalent and involved in the etiology of root caries, albeit to date, little has been explored regarding other microorganisms domains, such as archea, fungi and virus, and their role in biofilms. Previous studies demonstrated that Streptococcus mutans, Lactobacillus species (spp.), and Veillonella spp., as well as C. albicans, are present in major proportion in root caries than in sound root surface. ${ }^{2}$ Actinomyces spp., Veillonella spp., Streptococcus spp., Bifidobacterium spp., Rothia, Enterococcus, Staphylococcus spp., Capnocytophaga spp., Prevotella spp. and Candida spp., were also cultivated from root caries. ${ }^{1,3,4}$

Candida species has been associated with dental caries, especially with early childhood caries and root caries. ${ }^{5} \mathrm{~A}$ strong association was found between the prevalence of $C$. albicans and dental caries. ${ }^{6}$ Several authors showed that the proportion of Candida species was higher in individuals with caries than in individuals without caries. Furthermore, C. albicans is an important colonizer of carious lesions and has been found frequently in dentin caries lesions rather than in biofilm or saliva. ${ }^{4}$ Lower salivary flow rate, a common occurrence in older adults, is one of the factors that promote favorable conditions for a presence of $C$. albicans in these sites. ${ }^{7}$ However, it is still unknown whether the yeast acts as caries pathogen or plays a role as a commensal microbe. C. albicans possess some important properties that can characterize it as an important root caries pathogen. It is capable of adhering to saliva-coated hydroxyapatite and possesses strong adherence to collagen. ${ }^{8}$ It is as acid tolerant and acidogenic as S. mutans and Lactobacilli, which are both well-established cariogenic pathogens. ${ }^{9}$ To determine the role of $C$. albicans in root caries, a high-throughput sequencing of mRNA (RNA-Seq) was applied in clinical biofilms samples from two distinct conditions: sound root-surface biofilms and root carious lesions biofilms. This technique may be helpful to investigate Candida's role in a carious biofilm.

\section{Methodology}

This study is part of the project "metatranscriptome of root caries". ${ }^{10}$ Briefly, volunteers to this study were divided into two groups: sound exposed root surface group (SRS; $n=10$ ) and root caries group $(R C ; n=30)$. Participants were allocated to the SRS group $(n=10)$ if they had an exposed root surface on at least one tooth and no root caries lesions. Dental biofilms were collected with sterilized Gracey curette from all available exposed root surfaces. The number of exposed root surfaces varied among individuals. Participants recruited to the root caries (RC) group $(n=30)$ had one primary cavitated root lesion in need of restorative treatment. All lesions showed characteristics of present activity (soft and yellow dentin). Biofilm and carious dentin samples (of soft and infected tissue) were collected from patients during the restorative treatment. All participants were asked to refrain from tooth brushing for at least 12 hours prior to the sampling, to allow for dental biofilm accumulation, and were also asked to refrain from eating and drinking for at least 1 hour prior to the sampling. After collection, biofilm and carious dentin were immediately placed in $1 \mathrm{~mL}$ of RNA protect reagent (Qiagen, Hilden, North RhineWestphalia, Germany). The total RNA was extracted using the UltraClean ${ }^{\circledR}$ Microbial RNA Isolation (Mo-bio, San Diego, Califórnia, USA) with on-column DNase digestion (Qiagen, Hilden, North Rhine-Westphalia, Germany). Samples with total RNA concentration $<30$ ng/RNA were pooled, leading to a final sample size of 10 SRS and 9 RC. The Ribo-Zero ${ }^{\mathrm{TM}}$ Meta-Bacteria Kit (Illumina, Madison, Wisconsin, USA) was used for mRNA enrichment and Illumina ${ }^{\circledR}$ TruSeq $^{\mathrm{TM}}$ library prep protocols (Illumina, San Diego, Califórnia, USA) were used to library preparation and sequencing was performed with Illumina HiSeq2500 (Illumina, San Diego, Califórnia, USA). RNA sequencing data are available in the National Center for Biotechnology Information (NCBI) Sequence Read Archive, under the accession numbers SRS779973 and SRS796739. FASTQ files were obtained for each sample and imported into the CLC Genomics Workbench 7.5.1 software (CLC bio, Aarhus, Denmark) for mapping against 163 oral microbial genomes. ${ }^{10}$ The number of sequence reads that have been assigned to each gene is considered as the read count data. 


\section{Candida albicans genome and data analysis}

The C. albicans SC5314 was the genome selected for this study. This strain was chosen for being largely studied and its genome has been fully sequenced as well. After mapping, a count table was generated containing the read count for 14,217 oral C. albicans SC5314 genes.

The putative presence of the organism in the sample was estimated by the sum of reads assigned to $C$. albicans divided by the total number of genes for each sample. Samples with $\geq 14,217$ reads were considered as valid; then samples with less than $30 \%$ of genes with at least one read were excluded from the analysis.

The number of reads and the relative median expression (RME) $\left(25^{\text {th }}-75^{\text {th }}\right)$ level for genes were estimated for each of the sample groups, as previously described. ${ }^{11}$ Then, the RME was ranked to observe the most highly expressed transcripts in RC and SRS samples. To draw a profile of gene expression, the median of RME of transcripts in SRS and RC conditions were considered low expression RME between 0-10, medium 11-100, and high above 100 (percentile 10 of RME distribution). RME was calculated from the median values of normalized read counts using DESeq algorithm. Genes related to $C$. albicans virulence factors were analyzed: invasion, biofilm formation and co-aggregation, adherence and damage, morphogenesis, acid production, acid tolerance and stress response.

All RME medians for SRS and RC were ranked and all genes with median RME values $\geq 100$ per group were analyzed for an overview of the most prevalent genes.

Differential gene expression was inferred between sample groups by applying the R package DESeq2. ${ }^{12}$ The cut-off for designating a gene as being differentially expressed was a change in transcript levels of at least 2-fold change (Log2FoldChange $>1$ ) and false discovery rate $(F D R$ ) $<0.05$ (padj value $<0.05$, Benjamini \& Hochberg). Functions and putative pathogenicity in root caries of genes up-regulated in SRS and RC were analyzed.

Regarding to ethics considerations, this study was approved by the Federal University of Rio Grande do Sul research ethics committee (process $n^{\circ} 427.168$ ) and by the research ethics committee of the National Research Ethics Service Committee Yorkshire \& The Humber - Leeds West (protocol no. 2012002DD). All volunteers signed an informed consent form and received clinical dental assistance.

\section{Results}

According to the cut-off point chosen to determine the putative presence of a mapped organism in each sample, $C$. albicans was present in $n=4$ biofilms from SRS and in $n=6$ biofilm from RC, as shown in Figure 1 . Table 1 shows that the number of reads distribution in sound and disease samples were equal $(p=0.522)$.

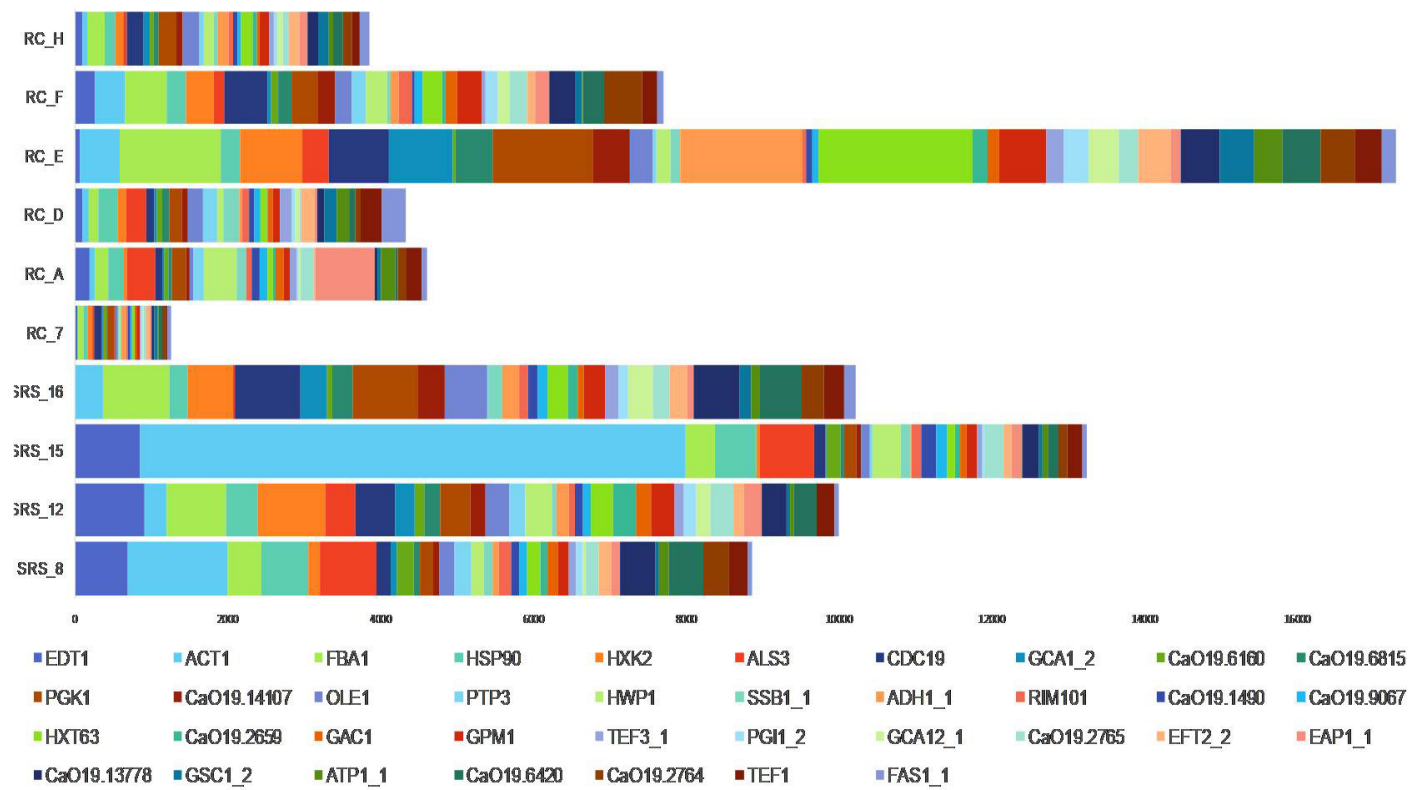

Figure 1- Relative median expression (RME; log10) of genes in the Sound Root Surfaces (SRS; $n=4$ ) and Root Caries (RC; $n=6$ ) samples. RME was calculated from the median values of normalized read counts. The top median RME values for SRS and RC were selected and sorted, and indicate the most expressed genes by C. albicans SC5413 
Table 1- Total numbers of DESeq normalized reads (median/ percentile/range) by group

\begin{tabular}{cccc}
\hline & Median & $\mathbf{2 5}^{\text {th }} \mathbf{- 7 5 ^ { \text { th } }}$ & Range \\
\hline SRS & 157.175 & $48.329-197.776$ & $22.721-223.511$ \\
RC & 209.495 & $79.770-571.876$ & $48.759-738.400$ \\
\hline
\end{tabular}

*SRS =sound root surface. $\mathrm{RC}=$ root caries

\section{Gene expression per sample}

Figure 1 shows an overview of the most prevalent genes in C. albicans biofilm with and without caries. A total of 37 genes with median of RME $>100$ were analyzed (34 in SRS and 20 in RC). A total of 17 genes have RME>100 in both health and disease conditions for all the samples (FBA1, HSP90, HXK2, ALS3, CDC19, PGK1, OLE1, HWP1, HXT63, GPM1, CaO19.2765, EFT2_2, EAP1_1, CaO19.13778, CaO19.6420, CaO19.2764 and TEF1), wheras 17 genes were expressed only in SRS (EDT1, ACT1, GCA1_2, CaO19.6160, CaO19.6815, CaO19.14107, PTP3, SSB1_1, ADH1_1, RIM101, CaO19.1490, CaO19.9067, CaO19.2659, GAC1, TEF3_1, PGI1_2 and GCA12_1) and just 3 genes were expressed only in RC conditions (GSC1_2, ATP1_1 and FAS1_1), as shown in Table 2.

\section{Expression of genes related to possible cariogenic traits}

C. albicans genes associated with possible virulence factors (RME and percentiles of these genes) were evaluated in both conditions (Table 2 ). We found transcript of 51 out of 67 genes related to virulence traits that are presented in the literature as important factors. None of these genes had significant differential expression.

\section{Differential expression analysis (DE)}

The DE analysis has shown the overexpressed genes in root biofilms with and without caries (Figure 2 ). The up-regulated genes in SRS group were $\mathrm{CaO} 19.610$ and CaO19.2506. The CaO19.610 (FDR=0.009) codes for a potential DNA binding regulator of filamentous growth. The CaO19.2506 (FDR=0.018) codes for a hypothetical protein with a very weak similarity to Streptococcal proline-rich surface protein PspC.

The up-regulated genes in RC group were UTP20, ITR1, DHN6, CaO19.7197, CaO19.7838, STT4, and GUT1. The UTP20 (FDR=0.018) codes for a potential U3 small nucleolar RNAs (snoRNA) protein. The ITR1 (FDR=0.036) codes for a potential active sugar transporter, potential Myo-inositol transporter, similar to S. cerevisiae ITR1 (YDR497C). The DHN6 $(F D R=0.046)$ codes for a dehydrin hypothetical protein. The CaO19.7197 (FDR=0.046) codes for a hypothetical protein similar to S. cerevisiae YLR002C, with unknown function. The $\mathrm{CaO} 19.7838(\mathrm{FDR}=0.046)$ codes for a flocculin-like protein serine-rich, repetitive ORF similar to S. cerevisiae MUC1 (YIR019C) cell surface flocculin. The STT4 (FDR=0.046) codes for a hypothetical protein phosphatidylinositol-4-kinase. The GUT1 (FDR=0.046) codes for a potential glycerol kinase Gut1p, likely carbohydrate kinase similar to $S$. cerevisiae GUT1 (YHL032C) glycerol kinase.

\section{Discussion}

Possible virulence traits of Candida spp. were related to several survival strategies such as the capacity to exploit and invade the host tissues, forming biofilms and co-aggregate to various microorganisms, switching form, producing acids and reacting to stress. C. albicans is metabolically active in biofilm of SRS and $\mathrm{RC}$, presenting different roles in health and disease. Some genes were expressed in both conditions, which seem to be relevant to $C$. albicans survival to these sites. Genes overexpressed in SRS were involved in biofilm formation, while genes overexpressed in RC were involved in survival strategies that could be related to cariogenicity.

Two genes were up-regulated in SRS biofilms. The CaO19.610 codes for a potential DNA binding regulator of filamentous growth. This gene is a version of $C$. albicans efg 1 with altered $C$ terminus. EFG1 protein is a key transcriptional regulator in C. albicans and controls various aspects of morphogenesis and metabolism ${ }^{13}$, being required for the true hyphae growth, biofilm formation, cell adhesion and filamentous growth in C. albicans. ${ }^{14}$ Efg1 gene confers to C. albicans the capacity of transition from commensal microorganism to opportunistic pathogen status. ${ }^{15}$ In an in vitro experiment, efg 1 had significantly higher gene expression at initial biofilm formation stage. ${ }^{16}$ Other studies showed that EFG1 is essential for the formation of a mature and stable biofilm that is resistant to antifungal therapy and to immune system, allowing the colonization of the root site. ${ }^{17,18}$ The $\mathrm{CaO} 19.2506$ codes for a hypothetical protein with a very weak similarity to streptococcal proline-rich surface protein PspC. In S. pneumoniae, PspC has a well-established importance 
Table 2- Relative median expression (RME) and percentiles $\left(25^{\text {th }}-75^{\text {th }}\right)$ of genes related to virulence factors in Candida albicans in the Sound Root Surfaces (SRS; $n=4)$ and Root Caries (RC; $n=6$ ) samples

\begin{tabular}{|c|c|c|c|}
\hline Accession ID & Median SRS $\left(25^{\text {th }}-75^{\text {th }}\right)$ & Median RC $\left(25^{\text {th }}-75^{\text {th }}\right)$ & Virulence Trait \\
\hline \multicolumn{4}{|l|}{ ACT } \\
\hline ACT1_1 & $416.13(156.10-2871.60)$ & $38.92(2.07-221.80)$ & \multirow{4}{*}{ Invasion } \\
\hline ACT1_2 & $415.74(147.50-2811.20)$ & $37.65(2.06-209.30)$ & \\
\hline ACT2_1 & $3.75(0.93-3.85)$ & $8.21(2.82-12.75)$ & \\
\hline ACT2_2 & $5.02(4.02-9.45)$ & $6.81(6.69-10.22)$ & \\
\hline \multicolumn{4}{|l|}{ LIP9 } \\
\hline LIP9_2 & $0(0-0)$ & $0.47(6.36-1.85)$ & \multirow{2}{*}{ Invasion } \\
\hline LIP9_1 & $0(0-0)$ & $0(6.10-1.22)$ & \\
\hline \multicolumn{4}{|l|}{ PLB⿳亠一冖 } \\
\hline PLB2_1 & $0(0-0.47)$ & $0.95(0-7.49)$ & \multirow{2}{*}{ Invasion } \\
\hline PLB2_2 & $0(0-0)$ & $0.18(0-4.16)$ & \\
\hline \multicolumn{4}{|l|}{ TEC1 $^{-}$} \\
\hline TEC1_1 & $21.98(18.5-26.30)$ & $14.46(2.65-26.40)$ & \multirow{2}{*}{ Biofilm Formation } \\
\hline TEC1_2 & $18.23(6.75-21.38)$ & $12.08(6.96-37.16)$ & \\
\hline EFG1 & $70.10(38.95-86.02)$ & $22.14(0-62.93)$ & Biofilm Formation/ Morphogenesis \\
\hline \multicolumn{4}{|l|}{ HWP1 } \\
\hline HWP1_1 & $127.29(19.59-176.94)$ & $80.53(0-167.24)$ & \multirow{2}{*}{ Biofilm Formation/ Adherence } \\
\hline HWP1_2 & $126.31(20.02-188.10)$ & $72.18(0-148.94)$ & \\
\hline \multicolumn{4}{|l|}{ ALS1 } \\
\hline ALS1_1 & $9.26(0.78-36.07)$ & $26.58(0-84.96)$ & \multirow[b]{2}{*}{ Adherence } \\
\hline ALS1_2 & $39.03(13.44-64.22)$ & $22.85(0-147.17)$ & \\
\hline \multicolumn{4}{|l|}{ ALS2 } \\
\hline ALS2_1 & 4.61(0.93-13.47) & $4.64(0.78-6.23)$ & \multirow{2}{*}{ Adherence } \\
\hline ALS2_2 & $2.79(0.62-9.47)$ & $3.73(0-7.46)$ & \\
\hline \multicolumn{4}{|l|}{ ALS3 } \\
\hline ALS3_1 & $199.32(45.47-274.46)$ & $88.26(0-122.72)$ & \multirow{5}{*}{ Invasion/Adherence } \\
\hline ALS3_2 & $50.15(3.16-98.69)$ & $33.79(0-77.51)$ & \\
\hline ALS3_3 & $171.47(34.94-255.39)$ & $91.19(2.51-124.35)$ & \\
\hline ALS3_4 & $159.55(34.17-225.69)$ & $24.39(1.47-89.82)$ & \\
\hline ALS4 & $5.93(1.04-13.48)$ & $10.63(0-23.22)$ & \\
\hline \multicolumn{4}{|l|}{ ALS5 } \\
\hline ALS5_1 & $3.47(2.07-4.99)$ & $5.04(0-11.62)$ & \multirow{3}{*}{$\begin{array}{l}\text { Adherence } \\
\text { Adherence }\end{array}$} \\
\hline ALS5_2 & $1.35(0.16-3.42)$ & $5.65(0-12.03)$ & \\
\hline ALS6 & $2.76(0-6.47)$ & $9.35(0-17.69)$ & \\
\hline ALS7 & $9.38(6.41-37.42)$ & $18.73(0-36.87)$ & Adherence \\
\hline ALS9 & & & \\
\hline ALS9_1 & $3.80(0-11.14)$ & $4.03(0-13.27)$ & \\
\hline ALS9_2 & $6.48(1.35-9.75)$ & $2.30(0-8.02)$ & Adherence \\
\hline ALS9_3 & $0(0-0)$ & $1.80(0-4.62)$ & \\
\hline RBT5 & & & \\
\hline RBT5_1 & $51.14(18.72-76.96)$ & $38.22(0-70.20)$ & \\
\hline RBT5_2 & $43.62(15.26-72.99)$ & $33.44(0-74.34)$ & Adherence \\
\hline SAP1 & & & \\
\hline SAP1_1 & $0(0-0.27)$ & $1.30(0-2.84)$ & \\
\hline SAP1_2 & $0(0-0.52)$ & $0(4.06-2.01)$ & Collagen degradation \\
\hline SAP2 & & & \\
\hline SAP2_1 & $0.31(0-1.19)$ & $0.93(55.86-4.55)$ & \\
\hline SAP2_2 & $0.35(0-4.2)$ & $1.18(115.19-4.03)$ & Collagen degradation \\
\hline SAP ${ }^{-}$ & & & \\
\hline SAP3_1 & $0(0-0)$ & $0(26.17-1.93)$ & \\
\hline SAP3_2 & $0(0-0.27)$ & $0.36(29.39-2.27)$ & Collagen degradation \\
\hline SAP4 & & & \\
\hline SAP4_1 & $0(0-0)$ & $0(0-0.61)$ & \\
\hline SAP4_2 & $0(0-0)$ & $1.65(0-4.38)$ & Collagen degradation \\
\hline SAP5 & & & \\
\hline SAP5_1 & $0(0-0.27)$ & $0.41(1.16-3.76)$ & on dearadation \\
\hline SAP5_2 & $0(0-0)$ & $1.85(0-3.56)$ & Collagen degradation/ Biotilm formation/Invasion \\
\hline
\end{tabular}

Continued on the next page 
Continued from previous page

\begin{tabular}{|c|c|c|c|}
\hline \multicolumn{4}{|l|}{ SAP6 } \\
\hline SAP6_1 & $0(0-0)$ & $0.59(0.52-3.61)$ & \multirow{3}{*}{ Collagen degradation } \\
\hline SAP6_2 & $0(0-0)$ & $1(0-6.06)$ & \\
\hline \multicolumn{3}{|l|}{ SAP7 } & \\
\hline SAP7_1 & $16.52(14.19-21.6)$ & $11.59(6.80-28.20)$ & \multirow{3}{*}{ Collagen degradation } \\
\hline SAP7_2 & $18.89(6.9-25.58)$ & $15.71(0-25.99)$ & \\
\hline \multicolumn{3}{|l|}{ SAP8 } & \\
\hline SAP8_1 & $0(0-4.03)$ & $1.295(0-4.85)$ & \multirow{3}{*}{ Collagen degradation } \\
\hline SAP8_2 & $0(0-0)$ & $0.06(0-3.67)$ & \\
\hline \multicolumn{3}{|l|}{ SAP9 } & \\
\hline SAP9_1 & $13.75(5.16-18.51)$ & $16.32(0.54-25.66)$ & \multirow{3}{*}{ Collagen degradation } \\
\hline SAP9_2 & $16.27(5.08-25.63)$ & $17.15(1.07-30.05)$ & \\
\hline \multicolumn{3}{|l|}{ SAP10 } & \\
\hline SAP10_1 & $0.31(0-0.94)$ & $1.84(4.84-6.65)$ & \multirow{3}{*}{ Collagen degradation } \\
\hline SAP10_2 & $0(0-0.27)$ & $1.06(13.5-3.99)$ & \\
\hline \multicolumn{3}{|l|}{ SAP98 } & \\
\hline SAP98_1 & $0.31(0-0.94)$ & $0.52(0.35-2.27)$ & \multirow{3}{*}{ Collagen degradation } \\
\hline SAP98_2 & $0(0-0)$ & $0.53(1.92-1.64)$ & \\
\hline \multicolumn{3}{|l|}{ SAP99 } & \\
\hline SAP99_1 & $0(0-0)$ & $1.24(0-4.56)$ & \multirow{3}{*}{ Collagen degradation } \\
\hline SAP99_2 & $0.18(0-1.01)$ & $0.12(0-1.93)$ & \\
\hline \multicolumn{3}{|l|}{ CDC24 } & \\
\hline CDC24_1 & $6.25(1.13-13.59)$ & $9.45(1.21-13.71)$ & \multirow{3}{*}{ Morphogenesis } \\
\hline CDC24_2 & $6.66(4.24-11.23)$ & $5.8(0-12.67)$ & \\
\hline \multicolumn{3}{|l|}{ CDC42 } & \\
\hline CDC42_1 & $6.58(1.99-11.37)$ & $3.45(0-4.37)$ & \multirow{3}{*}{ Morphogenesis } \\
\hline CDC42_2 & $1.38(0-3)$ & $2.95(0-3.9)$ & \\
\hline STE11 & & & \\
\hline STE11_1 & $9.07(5.47-17.16)$ & $7.33(0.54-14.34)$ & \\
\hline STE11_2 & $14.44(4.44-28.53)$ & $7.30(2.34-18.04)$ & Morphogenesıs \\
\hline CST20 & & & \\
\hline CST20_1 & $16.77(8.48-24.6)$ & $12.58(0-23.91)$ & \\
\hline CST20_2 & $16.99(12.72-21.04)$ & $15.66(0-22.24)$ & Morphogenesis \\
\hline HST7 & & & \\
\hline HST7_1 & $6.02(5.50-7.33)$ & $4.55(0-11.04)$ & \\
\hline HST7_2 & $5.06(1.24-5.33)$ & $3.73(0-9.39)$ & Morphogenesis \\
\hline CYR1 & & & \\
\hline CYR1_1 & $5.06(1.24-9.98)$ & $4.47(0-13.31)$ & Mornherenecis \\
\hline CYR1_2 & $8.35(5.96-10.10)$ & $7.98(0-17.23)$ & IVIorpnogenesis \\
\hline TPK2 & & & \\
\hline TPK2_1 & $6.71(5.57-7.61)$ & $5.79(0-9.88)$ & \\
\hline TPK2_2 & $5.64(1.13-7.49)$ & $7.98(0-10.83)$ & Morphogenesis \\
\hline PKA1 & & & \\
\hline PKA1_1 & $1.85(0-3.82)$ & $2.04(24.88-3.86)$ & \\
\hline PKA1_2 & $15.85(8.48-21.81)$ & $6.53(15.73-9.34)$ & Morphogenesis \\
\hline CZF1 & & & \\
\hline CZF1_1 & $0.52(0-2.11)$ & $1.27(5.05-5.85)$ & \\
\hline CZF1_2 & $1.75(0.26-4.65)$ & $1.35(6.26-3.44)$ & Morphogenesis \\
\hline NRG1 & $2.28(0.64-4.99)$ & $6.05(6.01-13.69)$ & Morphogenesis \\
\hline CPH1 & & & \\
\hline CPH1_1 & $9.58(1.56-35.08)$ & $8.13(0-17.53)$ & \\
\hline CPH1_2 & $0(0-0.47)$ & $0.59(0-1.48)$ & Morphogenesıs \\
\hline CDC28 & & & \\
\hline CDC28_1 & $0.35(0-1.56)$ & $1.69(0-3.16)$ & \\
\hline CDC28_2 & $1.04(0-8.58)$ & $2.83(0-6.96)$ & IVIorphogenesis \\
\hline CPH2 & & & \\
\hline $\mathrm{CPH}_{2} \_1$ & $52.42(21.47-56.66)$ & $23.81(0-46.74)$ & \\
\hline $\mathrm{CPH}_{2} \_2$ & $26.53(14.35-43.96)$ & $13.48(0-20.62)$ & Morphogenesis \\
\hline HSP90 & & & \\
\hline
\end{tabular}

Continued on the next page 
Continued from previous page

\begin{tabular}{|c|c|c|c|}
\hline HSP90_1 & 212.35(129.29-284.69) & $89.73(19.53-129.49)$ & \multirow{2}{*}{ Morphogenesis/ Stress Response } \\
\hline HSP90_2 & $254.89(158.85-313.28)$ & $111.99(14.61-143.75)$ & \\
\hline \multicolumn{4}{|l|}{ RAS1 } \\
\hline RAS1_1 & $2.59(0-5.46)$ & $5.45(0-6.90)$ & \multirow{2}{*}{ Morphogenesis } \\
\hline RAS1_2 & $3.30(0.62-5.07)$ & $4.68(0-5.76)$ & \\
\hline \multicolumn{4}{|l|}{ TUP1 } \\
\hline TUP1_1 & 24.91(19.85-32.21) & $10.47(0-14.82)$ & \multirow{2}{*}{ Morphogenesis } \\
\hline TUP1_2 & $20.69(9.82-28.32)$ & $8.03(0-10.93)$ & \\
\hline \multicolumn{4}{|l|}{ RFG1 } \\
\hline RFG1_1 & $0(0-1.04)$ & $2.28(0-6.62)$ & \multirow{3}{*}{ Morphogenesis } \\
\hline RFG1_2 & $7(1.35-8.9)$ & $5.59(0-11.98)$ & \\
\hline \multicolumn{3}{|l|}{ HYR1 } & \\
\hline HYR1_1 & $0(0-0.47)$ & $0.47(0-1.67)$ & \multirow{3}{*}{ Morphogenesis } \\
\hline HYR1_2 & $0(0-0.47)$ & $1.42(0-7.26)$ & \\
\hline \multicolumn{3}{|l|}{ ECE1 } & \\
\hline ECE1_1 & $0.18(0-2.40)$ & $0.42(0-1.93)$ & \multirow{2}{*}{ Morphogenesis } \\
\hline ECE1_2 & $1.23(0-4.65)$ & $1.03(0-4.44)$ & \\
\hline \multicolumn{4}{|l|}{ PHR1 } \\
\hline PHR1_1 & $33.62(25.20-94.04)$ & $38.18(0-57.07)$ & \multirow{2}{*}{ Acid tolerance } \\
\hline PHR1_2 & $36.96(27.23-104.02)$ & $48.26(0-57.07)$ & \\
\hline \multicolumn{4}{|l|}{ PHR2 } \\
\hline PHR2_1 & $4.71(2.27-29.58)$ & $7.77(0.78-32.06)$ & \multirow{2}{*}{ Acid tolerance } \\
\hline PHR2_2 & $51.11(2.69-118.06)$ & $58.10(0.18-137.34)$ & \\
\hline RIM101 & $120.39(89.45-151.32)$ & $65.67(2.72-111.54)$ & Stress response/ Morphogenesis \\
\hline \multicolumn{4}{|l|}{ HOG1 } \\
\hline HOG1_1 & $4(1.89-5.07)$ & $4.36(1.30-5.09)$ & \multirow{2}{*}{ Stress response } \\
\hline HOG1_2 & $3.96(0.77-6.99)$ & $4.2(1.065-7.63)$ & \\
\hline \multicolumn{4}{|l|}{ CAP1 } \\
\hline CAP1_1 & 7.15(1.73-7.64) & $5.21(3.12-8.725)$ & \multirow{2}{*}{ Stress response } \\
\hline CAP1_2 & $11.01(6.34-14.78)$ & $9.25(1.65-11.7)$ & \\
\hline
\end{tabular}

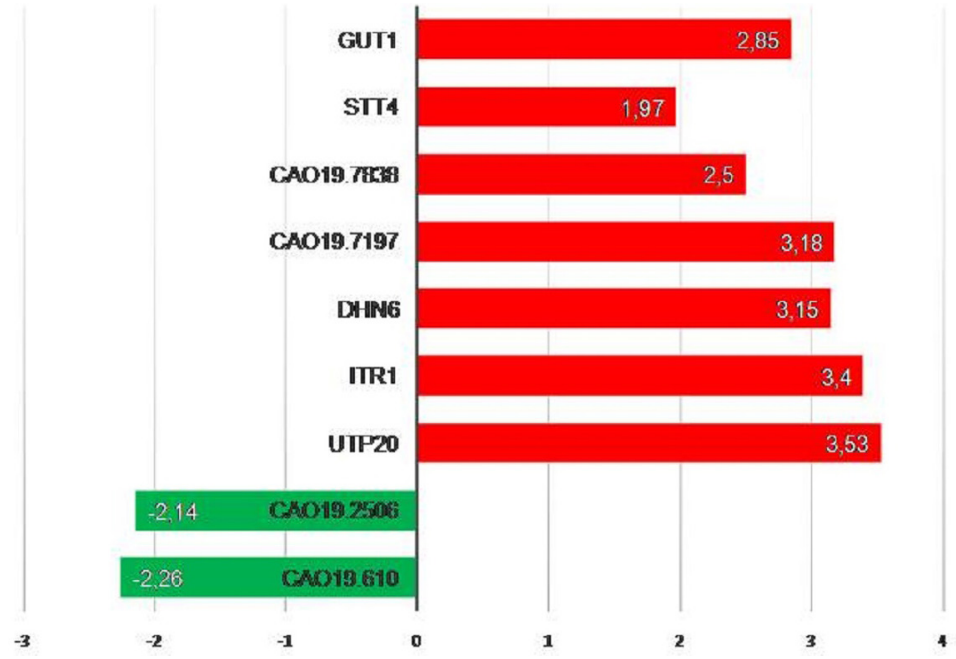

Figure 2- Differential expression (Log2FoldChange) of genes up-regulated in sound root surface (SRS; negative values, green bars) and up-regulated in root caries (RC, positive values, red bars) calculated using DESeq2 algorithms. FDR $<0.05$. GUT1= potential glycerol kinase; STT4= hypothetical protein phosphatidylinositol-4-kinase; CaO19.7838= flocullin-like protein serine-rich; CaO19.7197= hypothetical protein; DHN6= dehydrin hypothetical protein; ITR1= potential Myo-inositol transporter; UTP20= potential U3 small nucleolar RNAs protein; $\mathrm{CaO} 19.2506=$ hypothetical protein with a very weak similarity to Streptoccal proline-rich surface protein $\mathrm{PspC} ; \mathrm{CaO} 19.610=$ potential DNA binding regulator of filamentous growth

in adherence and colonization. ${ }^{19}$ The possible function of $\mathrm{CaO} 19.2506$ is related to adhesion and coding for a membrane adhesin. Both filamentous growth and cell wall adhesion are important in biofilm formation and are required for the establishment of $C$. albicans in root surfaces biofilm. These characteristics could explain why $C$. albicans has been largely observed colonizing sound root surface. ${ }^{5}$

The role of $C$. albicans in root caries could be potentially more complex. Seven genes were upregulated in root caries conditions expressing different functions. The CaO19.7197 codes for a hypothetical 
protein similar to S. cerevisiae YLR002C, with unknown function. Several hypothetical proteins and genes with uncharacterized function were identified in this study, highlighting the importance of more studies related to $C$. albicans transcriptome. The DHN6 codes for a dehydrin hypothetical protein, related to stress tolerance in plants. These proteins can be induced in vegetative tissues by different stress factors that cause cell dehydration (i.e., drought, salinity, cold, heat, low temperature, etc)..$^{20,21}$ The STT4 codes for a hypothetical protein phosphatidylinositol-4-kinase. The gene STT4 is essential for viability and plays an important role in the phosphatidylinositol-mediated signal transduction pathway required for cell wall integrity. ${ }^{22}$ Therefore, the up-regulated genes DHN6 and STT4 could be related to the ability to survive in an extreme environment with several stress factors (low pH, carbohydrate viability, for example) such as the ones found in root cavitated caries lesions. The UTP20 codes for a potential U3 small nucleolar RNAs (snoRNA) protein. UTP20 has been reported as a component of U3 snoRNA protein complex and has been implicated in 18S rRNA processing, being essential for 18 rRNA function. ${ }^{23,24}$ The ITR 1 codes for a potential active sugar transporter, potential Myo-inositol transporter, similar to $S$. cerevisiae ITR1 (YDR497C). Myo-inositol is an essential substrate for C. albicans, and it can be used as carbon source. For its survival, C. albicans must be able to synthesize the essential metabolite inositol or acquire it from the host. C. albicans could not transport inositol and become nonviable in the absence of ITR 1.25 The CaO19.7838 codes for flocculin-like protein serine-rich, repetitive ORF similar to S. cerevisiae MUC1 (YIR019C) cell surface flocculin. MUC1 encodes cell-surface flocculin and it is required for pseudohyphal and invasive growth of C. albicans. ${ }^{14}$ The up-regulation of this invasive growth gene shows the importance of this virulence trait for the colonization/penetration of $C$. albicans in the carious dentin. The GUT1 codes for a potential glycerol kinase Gut1p, likely carbohydrate kinase similar to S. cerevisiae GUT1 (YHL032C) glycerol kinase (NCBI). In Saccharomyces cerevisiae, glycerol utilization is mediated by two enzymes, glycerol kinase (Gut1p) and mitochondrial glycerol-3-phosphate dehydrogenase (Gut2p). The carbon source regulation of GUT1 depends on carbon source availability. The promoter activity of GUT1 was lower during growth on glucose and highest on the non-fermentable carbon sources, glycerol, ethanol, lactate, acetate and oleic acid. ${ }^{26}$ UTP20, ITR1, CaO19.7838, and GUT1 are genes related to $C$. albicans metabolism associated with caries progression to a cavitated status.

The overexpressed genes in RC were related to sugar transport (ITR1 - Myo-inositol) and to carbon source regulation (GUT1 - Glycerol kinase), that were related to the use of alternative carbon sources (Figure 2 ). The use of lactate by $C$. albicans could be related to the $\mathrm{pH}$ regulation (neutralization) in biofilm, which is important for the microbiome survival. ${ }^{27}$ Furthermore, this neutralization of the medium could explain the $\mathrm{CaO} 19.7838$ overexpression, a gene related to hyphal growth since hyphal formation is stimulated by neutral $\mathrm{pH} .{ }^{28}$ Morphogenesis is a special virulence trait of C. albicans, and hyphal form is related to pathogenesis, being more invasive and contributing to host tissue damage ${ }^{29,30}$, as well as contributing to the active cavitation of RC lesions. Besides the stimulation of hyphal growth, changes in carbon source has a significant impact on the C. albicans virulence, resulting in an increased resistance to stresses, adherence, biofilm formation, drug resistance, and immune recognition when compared with glucosegrown cells. ${ }^{31}$ Although a cariogenic environment is related to low $\mathrm{pH}$ conditions, the excessive production of acids could affect the biofilm metabolism. For the cariogenic biofilm survival, it is important to have a microorganism that main the viability of the biofilm, thus preventing excessive acidification even in a carious habitat.

\section{Conclusions}

Our data shows that Candida albicans SC5314 have an active metabolism in biofilm of SRS and biofilm of carious dentin of RC as well. The differential expression analysis shows that, in healthy individuals, the upregulated genes were related to metabolic activity, sugar transport, stress tolerance, invasion and $\mathrm{pH}$ regulation. $C$. albicans may have a role in root caries progression.

\section{Conflicts of interest statement}

The authors declare no conflicts of interest in the subject matter discussed in this manuscript. 


\section{Acknowledgements}

The authors would like to thank Brazilian National Counsel of Technological and Scientific Development (CNPQ) (process no. 482504/2013-7), Coordination for the Improvement of Higher Level Education (CAPES) (process no 18097-12-0), Rio Grande do Sul State Foundation for Research Support (FAPERGS) (process no. 001/2013 PQG) and Leeds Teaching Hospitals Charitable Foundation (R\&D/PP/12011), Dunhill Medical Trust (R245/0212). This study is derived from a thesis submitted by the first author to the Dental School of Federal University of Rio Grande do Sul as partial fulfilment of the requirements for a master's degree in Clinical Dentistry - Cariology/ Operative Dentistry. Partial results were presented at the $65^{\text {th }}$ ORCA Congress, held in Copenhagen (Denmark), 2018.

\section{Authors' Contributions}

Ev, Laís Daniela: Data curation (Equal); Formal analysis (Equal); Resources (Equal); Software (Equal); Visualization (Equal); Writing-original draft (Equal); Writing-review \& editing (Equal); Damé-Teixeira, Naile: Conceptualization (Equal); Data curation (Equal); Formal analysis (Equal); Methodology (Equal); Project administration (Equal); Resources (Equal); Validation (Equal); Writing-original draft (Equal); Do, Thuy: Data curation (Equal); Formal analysis (Equal); Funding acquisition (Equal); Methodology (Equal); Project administration (Equal); Supervision (Equal); Writingoriginal draft (Equal); Maltz, Marisa: Conceptualization (Equal); Formal analysis (Equal); Funding acquisition (Equal); Project administration (Equal); Supervision (Equal); Writing-original draft (Equal); Parolo, Clarissa: Conceptualization (Equal); Data curation (Equal): Formal analysis (Equal); Investigation (Equal); Methodology (Equal); Supervision (Equal); Validation (Equal); Visualization (Equal); Writingoriginal draft (Equal) Writing-review \& editing (Equal).

\section{References}

1- Brailsford SR, Shah B, Simons D, Gilbert S, Clark D, Ines I, et al. The predominant aciduric microflora of root-caries lesions. J Dent Res. 2001;80(9):1828-33.

2- Marchant S, Brailsford SR, Twomey AC, Roberts GJ, Beighton D. The predominant microflora of nursing caries lesions. Caries Res. 2001;35(6):397-406. doi: 10.1159/000047482
3- Shen S, Samaranayake LP, Yip HK, Dyson JE. Bacterial and yeast flora of root surface caries in elderly, ethnic Chinese. Oral Microbiol. 2002;8(4):207-17. doi: 10.1034/j.1601-0825.2002.01796.x

4- Mantzourani M, Fenlon M, Beighton D. Association between Bifidobacteriaceae and the clinical severity of root caries lesions. Oral Microbiol Immunol. 2009;24(1):32-7. doi: 10.1111/j.1399302X.2008.00470.x

5- Carvalho FG, Silva DS, Hebling J, Spolidorio LC, Spolidorio DM. Presence of mutans streptococci and Candida spp. in dental plaque/ dentin of carious teeth and early childhood caries. Arc Oral Biol. 2006;51(11):1024-8. doi: 10.1016/j.archoralbio.2006.06.001 6- Beighton D, Brailsford S, Samaranayake LP, Brown JP, Ping FX, Grant-Mills D, et al. A multi-country comparison of caries-associated microflora in demographically diverse children. Community Dent Health. 2004;21(1):96-101

7- Do T, Damé-Teixeira N, Naginyte M, Marsch PD Root surface biofilms and caries. Monogr Oral Sci. 2017;26:26-34. doi: 10.1159/000479304 8- Makihira S, Nikawa H, Tamagami M, Hamada T, Samaranayake LP. Differences in Candida albicans adhesion to intact and denatured type I collagen in vitro. Oral Microbiol Immunol. 2002;17(2):129-31. doi: 10.1046/j.0902-0055.2001.00094.x

9- Aas JA, Griffen AL, Dardis SR, Lee AM, Olsen I, Dewhirst FE, et al. Bacteria of dental caries in primary and permanent teeth in children and young adults. J Clin Microbiol. 2008;46(4):1407-17. doi: 10.1128/ JCM.01410-07

10- Damé-Teixeira N, Parolo CC, Maltz M, Tugnait A, Devine D, Do T. Actinomyces spp. gene expression in root caries lesions. J Oral Microbiol. 2016;6(8):32383. doi: 10.3402/jom.v8.32383

11- Do T, Sheehy EC, Mulli T, Hughes F, Beighton D. Transcriptomic analysis of three Veillonella spp. present in carious dentin and in the saliva of caries-free individuals. Front Cell Infect Microbiol. 2015;5:25. doi: $0.3389 /$ fcimb. 2015.00025

12- Love M, Anders S, Huber W. Differential analysis of count data - the DESeq2 package [Internet]. 2016 Nov 30. [cited 2019 Oct 1]. Available from: https://www.genomatix.de/online_help/help_regionminer/ DESeq2.pdf

13- Noffz CS, Liedschulte V, Lengeler K, Ernst JF. Functional mapping of the Candida albicans Efg1 regulator. Eukaryotic Cell. 2008;7(5):88193. doi: doi: 10.1128/EC.00033-08

14- Gancedo JM. Control of pseudohyphae formation in Saccharomyces cerevisiae. FEMS Microbiology Reviews. 2001;25(1):107-23. doi: 10.1111/j.1574-6976.2001.tb00573.x

15- Pierce VJ, Kumamoto AC. Variation in Candida albicans EFG1 expression enables host-dependent changes in colonizing fungal populations. MBio. 2012;3(4):e00117-12. doi: 10.1128/mBio.0011712

16- Barros PP, Rossoni RD, Camargo Ribeiro F, Junqueira JC, Jorge AO. Temporal profile of biofilm formation, gene expression and virulence analysis in Candida albicans strains. Mycopathologia. 2017;182(34):285-95. doi: 10.1007/s11046-016-0088-2

17- Finkel JS, Mitchell AP. Genetic control of Candida albicans biofilm development. Nat Rev Microbiol. 2011;9(2):109-18. doi: 10.1038/ nrmicro2475

18- Nobile CJ, Fox EP, Nett JE, Sorrells TR, Mitrovich QM, Hernday $A D$, et al. A recently evolved transcriptional network controls biofilm development in Candida albicans. Cell. 2012;148(1-2):126-38. 19- Rosenow CP, Ryan JN, Weiser S, Johnson P, Fontan A, Ortqvist $H R$, et al. Contribution of novel choline-binding proteins to adherence, colonization, and immunogenicity of Streptococcus pneumoniae. Mol Microbiol. 1997;25(5):819-29. 10.1111/j.1365-2958.1997.mmi494.x 20- Hanin M, Brini F, Ebel C, Toda Y, Takeda S, Masmoudi K. Plant dehydrins and stress tolerance: versatile proteins for complex mechanisms. Plant Signal Behav. 2011;6(10):1503-9. doi: 10.4161/ psb.6.10.17088 
21- Drira M, Saibi W, Amara I, Masmoudi K, Hanin M, Brini F. Wheat dehydrin $\mathrm{K}$-segments ensure bacterial stress tolerance, antiaggregation and antimicrobial effects. Appl Biochem Biotechnol. 2015;175(7):331021. doi: 10.1007/s12010-015-1502-9

22- Pramanik A, Garcia E, Ospina R, Powell M, Martinez M, Alejo W, et al. Cloning, characterization and identification of the gene encoding phosphatidylinositol 4-kinase. Cell Mol Biol. 1997;43(7):1007-18.

23- Wang Y, Liu J, Zhao H, Lü W, Zhao J, Yang L, et al. Human 1 A6/DRIM, the homolog of yeast Utp20, functions in the $18 \mathrm{~S}$ rRNA processing. Biochim Biophys Acta. 2007;1773(6):863-8. doi: 10.1016/j.bbamcr.2007.04.002

24- Dez C, Dlakić M, Tollervey D. Roles of the HEAT repeat proteins Utp10 and Utp20 in 40S ribosome maturation. RNA. 2007;13(9):151627. doi: 10.1261/rna.609807

25- Chen YL, Kauffman S, Reynolds TB. Candida albicans uses multiple mechanisms to acquire the essential metabolite inositol during infection. Infect Immun. 2008;76(6):2793-801. doi: 10.1128/ IAI.01514-07

26- Grauslund M, Lopes JM, Rønnow B. Expression of GUT1, which encodes glycerol kinase in Saccharomyces cerevisiae, is controlled by the positive regulators Adr1p, Ino2p and Ino4p and the negative regulator Opi1p in a carbon source-dependent fashion. Nucleic Acids Res. 1999;27(22):4391-8. doi: 10.1093/nar/27.22.4391
27- Danhof HA, Vylkova S, Vesely EM, Ford AE, Gozalez-Garay M, Lorenz M. Robust extracellular $\mathrm{pH}$ modulation by Candida albicans during growth in carboxylic acids. mBio. 2016;7(6):e01646-16. doi: 10.1128/mBio.01646-16

28- Sudbery PE. Growth of Candida albicans hyphae. Nat Rev Microbiol. 2011;9(10):737-48. doi: 10.1038/nrmicro2636

29- Calderone RA, Fonzi WA. Virulence factors of Candida albicans. Trends Microbiol. 2001;9(7):327-35. doi: 10.1016/s0966-842x(01)02094-7 30- Höfs S, Mogavero S, Hube B. Interaction of Candida albicans with host cells: virulence factors, host defense, escape strategies, and the microbiota. J Microbiol. 2016;54(3):149-69. doi: 10.1007/ s12275-016-5514-0

31- Ene IV, Heilmann CJ, Sorgo AG, Walker LA, de Koster CG, Munro CA, et al. Carbon source-induced reprogramming of the cell wall proteome and secretome modulates the adherence and drug resistance of the fungal pathogen Candida albicans. Proteomics. 2012;12(21):3164-79. doi: 10.1002/pmic. 201200228 